\title{
Identification and Characterization of the Replication Region of Virulence Plasmid pEIB202 in Edwardsiella piscicida ${ }^{\mathrm{S}}$
}

\author{
Xinyue Chang ${ }^{1}$, Chengli Teng ${ }^{1}$, Haizhen $\mathrm{Wu}^{1,2 *}$, Jiang $\mathrm{Ye}^{1}$, Qiyao Wang ${ }^{1}$, and Huizhan Zhang ${ }^{1,2}$ \\ ${ }^{1}$ State Key Laboratory of Bioreactor Engineering, East China University of Science and Technology, Shanghai, P.R. China \\ ${ }^{2}$ Department of Applied Biology, East China University of Science and Technology, Shanghai, P.R. China
}

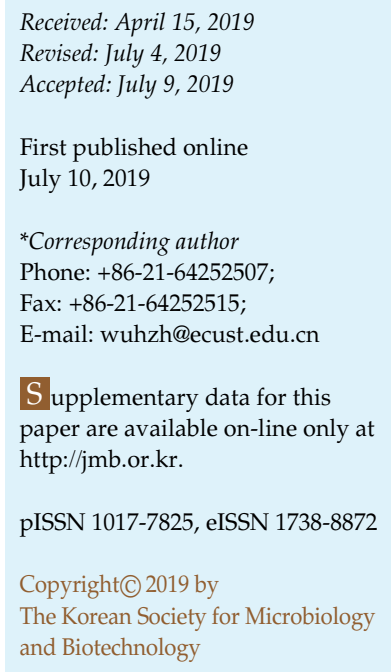

\begin{abstract}
Edwardsiella piscicida is the causative agent of edwardsiellosis, which has caused enormous economic losses worldwide. In our previous research, an attenuated live vaccine known as WED and based on the virulent strain E. piscicida EIB202 can effectively protect turbots against edwardsiellosis via intraperitoneal injection, while vaccination by immersion exhibits a weaker effect. During the development of the immersion vaccine, we surprisingly found the counts of $\Delta$ pEIB202/ EIB202 colonized on zebrafish were 100 times lower than those of EIB202. However, pEIB202 carries 53 predicted ORFs and has several copies in E. piscicida EIB202, impeding the study of its function. Thus, the replication region is located at a $1,980 \mathrm{bp}$ fragment (from 18,837 to 20,816 bp), containing a transcriptional repressor and a replication protein. Moreover, the minimal replication plasmid, named pRep-q77, has low copies in both E. coli and E. piscicida, but is more stable in E. piscicida than in E. coli. This work lays a foundation for further examination of the function of the virulence plasmid pEIB202.
\end{abstract}

Keywords: Edwardsialla piscicida, pEIB202, replication region, location, characterization

\section{Introduction}

Edwardsiella piscicida, a Gram-negative, short rod-shaped, motile, facultatively anaerobic bacterium [1], can cause edwarsiellosis characterized by symptoms of petechial hemorrhage, bloody ascites, exophthalmia, rectal hernia, and congested organs [2]. It has infected an enormous range of hosts, including fish, amphibians, reptiles, birds, and mammals [3]. Moreover, E. piscicida has been recognized to induce systematic disease in over 20 valuable farmed fish species, resulting in tremendous economic losses worldwide [4].

With the significant growth of aquaculture during the past five decades, the application of antibiotics has also increased dramatically, which induces drug resistance in bacteria or suppresses the immune system of host [5]. In addition, E. piscicida is insensitive to a majority of antibiotics since it is an intracellular pathogen. In this case, developing vaccines is an effective way to protect fish against edwarsiellosis. Several vaccines against various virulence factors including flagella [6], invasin [7], catalase [8], and two-component systems $[9,10]$ have been developed. Previously, we developed WED, a live attenuated vaccine, with deletion of aroC, TTSS, and pEIB202 of E. piscicida EIB202. It was found that the immunoprotective effect via immersion was lower than that of injection vaccination [11]. But since immersion is a labor-and-time-efficient method, improving the effectiveness of immersion vaccination is of significance to commercial application of this vaccine. Much work has been done on the development of immersion vaccines. And we surprisingly found the plasmid pEIB202 $(43,703 \mathrm{bp})$ in E. piscicida EIB202 could play an important role in its colonization on zebrafish.

Plasmid pEIB202 is an endogenous multi-drug resistance plasmid (GenBank Accession No. CP001136) in E. piscicida EIB202 [12]. The plasmid carries 53 ORFs, including antibiotic resistance genes, conjugation genes, and an incomplete set of a type IV secretion system. It also has more than one copy in EIB202 (not shown by data), hindering the deletion of genes on plasmid and examination of the function of this virulence plasmid. Even though the replication initiator protein was predicted [12], the exact locus of the replicon 
Table 1. Bacterial strains and plasmids used in this study.

\begin{tabular}{|c|c|c|}
\hline Strains and plasmids & Characteristics & Source \\
\hline \multicolumn{3}{|l|}{ Strains } \\
\hline E. coli JM83 & $\mathrm{F}^{-}$, ara, $\Delta(l a c-p r o \mathrm{AB}), \operatorname{rpsL},\left(\mathrm{Str}^{\mathrm{r}}\right), \phi 80, \Delta($ lacZ $), \mathrm{M} 15$ & Our lab \\
\hline E. piscicida EIB202 & $\mathrm{Col}^{\mathrm{r}}, \mathrm{Cm}^{\mathrm{r}}$, wild type & Our lab [13] \\
\hline$\Delta$ pEIB202/EIB202 & $\mathrm{Col}^{\mathrm{r}}$, pEIB202 curing derivative of EIB202 & Our lab \\
\hline WED & A live attenuated vaccine with deletion of aroC, TTSS, and pEIB202 of E. piscicida EIB202 & Our lab [11] \\
\hline \multicolumn{3}{|l|}{ Plasmids } \\
\hline pEIB202 & Endogenous plasmid in E. piscicida EIB202 & Our lab [12] \\
\hline pRep 33 & $\begin{array}{l}\text { Sau3A I partially digested fragment, located at } 18,249 \sim 21,335 \text { bp in pEIB202, ligated } \\
\text { with an } 892 \text { bp DNA fragment }\left(\mathrm{KCm}^{\mathrm{r}}\right) \text { containing Cm resistance gene. }\end{array}$ & This work \\
\hline pRep 23 & $\begin{array}{l}\text { Sau3A I partially digested fragment, located at } 17,961 \sim 21,335 \mathrm{bp} \text { in pEIB202, ligated } \\
\text { with an } 892 \mathrm{bp} \text { DNA fragment }\left(\mathrm{KCm}^{\mathrm{r}}\right) \text { containing Cm resistance gene. }\end{array}$ & This work \\
\hline pRep 19 & $\begin{array}{l}\text { Sau3A I partially digested fragment, located at } 18,134 \sim 21,602 \mathrm{bp} \text { in pEIB202, ligated } \\
\text { with an } 892 \mathrm{bp} \text { DNA fragment }\left(\mathrm{KCm}^{\mathrm{r}}\right) \text { containing Cm resistance gene. }\end{array}$ & This work \\
\hline pRep 13 & $\begin{array}{l}\text { Sau3A I partially digested fragment, located at 17,123 21,602 bp in pEIB202, ligated } \\
\text { with an } 892 \text { bp DNA fragment }\left(\mathrm{KCm}^{\mathrm{r}}\right) \text { containing Cm resistance gene. }\end{array}$ & This work \\
\hline pRep-q77 & $\begin{array}{l}\text { Plasmid with minimal replicon, located at } 18,837 \sim 20,816 \mathrm{bp} \text { in pEIB202, ligated with an } \\
892 \mathrm{bp} \text { DNA fragment }\left(\mathrm{KCm}^{\mathrm{r}}\right) \text { containing } \mathrm{Cm} \text { resistance gene. }\end{array}$ & This work \\
\hline pDMK & $\mathrm{Km}^{\mathrm{r}}, \mathrm{Cm}^{\mathrm{r}}$, pDM4 derivative, suicide vector, $\mathrm{R} 6 \mathrm{~K}$, pir requiring & Our lab [14] \\
\hline
\end{tabular}

has not been identified. The understanding of pEIB202 would be facilitated by adding specific genes to the replicon instead of deleting genes on plasmid. To this end, we successfully located and characterized the replication region of pEIB202, which lays a foundation for studying the virulent function of pEIB202.

\section{Materials and Methods}

\section{Strains, Plasmids and Growth Conditions}

Strains, plasmids, and primers used in this study are listed in Tables 1 and 2. E. piscicida strains were cultivated in Luria broth (LB) or LB with $1.8 \%$ agar at $28^{\circ} \mathrm{C}$. E. coli strains were cultivated in $\mathrm{LB}$ or LB with $1.8 \%$ agar at $37^{\circ} \mathrm{C}$. When required, chloramphenicol $(\mathrm{Cm})$ was added at $30 \mu \mathrm{g} / \mathrm{ml}$ right after inoculation.

\section{Plasmid DNA Isolation}

The isolation of plasmid pEIB202 from E. piscicida EIB202 was performed as described by Xi X et al. with some adjustments [15]. Strains were grown overnight and then collected by centrifugation and lysed in Buffer I ( $50 \mathrm{mM}$ glucose, $50 \mathrm{mM}$ Tris- $\mathrm{HCl}, 10 \mathrm{mM}$ EDTA, $\mathrm{pH}$ 8.0). Then lysozyme (final concentration: $5 \mathrm{mg} / \mathrm{ml}$ ) was added, after which the mixture was incubated at $37^{\circ} \mathrm{C}$ for $30 \mathrm{~min}$. Buffer II was then added and incubated at $37^{\circ} \mathrm{C}$ until the solution was clear, followed by addition of Buffer III and an icebath for $30 \mathrm{~min}$. A plasmid extraction kit (GK2002-200; Generay, China) was used to isolate plasmid DNA from E. coli. DNA products were stored at $-20^{\circ} \mathrm{C}$.

\section{E. piscicida Electroporation}

To transform plasmid to E. piscidida ApEIB202/EIB202, electroporation was carried out with glass cuvettes. Briefly, the electrocompetent bacteria were ice-bathed till glycerol melted and plasmid DNA was added and mixed evenly. Then the bacteriaDNA mixture was transferred to glass cuvette and the outside was kept dry. The cuvette was placed in an electroporator (MicroPulser, Bio-Rad, USA) and a $3 \mathrm{kV}, 6 \mathrm{~ms}$ pulse was performed. Afterwards, $1 \mathrm{ml}$ of pre-warmed LB media was pipetted into the cuvette immediately and mixed gently. Next, the bacteria were cultured at

Table 2. Primers used in this study.

\begin{tabular}{ll}
\hline $\begin{array}{c}\text { Primer } \\
\text { name }\end{array}$ & \multicolumn{1}{c}{ Nucleotide sequence $\left(5^{\prime}-3^{\prime}\right)$} \\
\hline Rep q77-F & CCCAAGCTTAATCAAAACACCCCATCTT \\
Rep q77-R & CGCGGATCCTGAGGCTTCGCCATTAGTAATAGGG \\
KCm-F & CGCGGATCCCGCACTTTGCGCCG \\
KCm-R & CGCGGATCCTTACGCCCCGCCCTGCCAC \\
Cm30 & ATTTATTCTGCGAAGTGATCTTCCG \\
Cm840 & TTCCATGTCGGCAGAATGCTTAATG \\
gap-F & AACATCATCCCGTCCTCTAC \\
gap-R & CCATACCAGTCAGTTTGCCA \\
hlyB-F & GCCTATTTCGTCTTTCCTAA \\
hlyB-R & ACATCGCTATCCATCCGTCT \\
rep-F & AGCTGCCACCGCTGTACAC \\
rep-R & CTTCCTGTCACGCTCCCTA \\
\hline
\end{tabular}


$28^{\circ} \mathrm{C}$ with $190 \mathrm{rpm}$ shaking for $2 \mathrm{~h}$, followed by plating the transformed bacteria to LB agar containing proper antibiotics.

\section{DNA Sequencing and Analysis}

Partially digested pEIB202 by Sau3A I were ligated to an 892 bp fragment encoding chloramphenicol resistance, which was amplified by $\mathrm{KCm}-\mathrm{F} / \mathrm{R}$ primers on $\mathrm{pDMK}$. The ligation products were then transformed into E. coli JM83. The locations of subclones were determined by aligning the sequence of each subclone with pEIB202. The sequencing was performed with $\mathrm{Cm} 30 / \mathrm{Cm} 480$ primers on $\mathrm{KCm}^{\mathrm{r}}$ fragment. Sequence analysis was performed by using the NCBI database or the DNASTAR (Lasergene.v 7.1, DNASTAR Inc) software. The phylogenetic NJ tree of RepA protein from pEIB202 was formed by clustering the top 15 hits after BLASTP similarity searches at Genbank. Then, the relationships were constructed by MEGA 7 .

\section{Determination of Plasmids Copy Number with qPCR Assays}

The copy number of the derivates of pEIB202 in E. coli and E. piscicida was measured by qPCR, as described previously [16]. A Bio-Rad CFX96 Real-time PCR System (Bio-Rad) was used for qPCR amplification and detection. The qPCR assay was performed in a $20 \mu$ reaction system in triplicate, with $10 \mu \mathrm{l}$ SYBR Green Real-Time PCR Master Mix (TOYOBO, Japan), $2 \mu$ l Plus solution (TOYOBO), $1 \mu \mathrm{l}$ forward primer, $1 \mu \mathrm{l}$ reverse primer, $1 \mu \mathrm{l}$ template and $5 \mu \mathrm{lddH_{2 }}$ O. Cultured E. coli and E. piscicida were added as template. PCR primers were designed by Primer Premier 5.0 software. The glyceraldehyde-3-phosphate dehydrogenase gene gap, a single-copy gene in the genome of E. coli, and hemolysin activator HlyB domain gene hlyB [17], single-copy in E. piscicida, were used as the reference genes. The rep- $\mathrm{F} / \mathrm{R}$ were designed for detection of pEIB202 derivates. The copy counts of plasmids were calculated as follows: $\mathrm{N}=2^{-\Delta \mathrm{Ct}} . \Delta \mathrm{Ct}$ represented the difference between the threshold cycle counts $(\mathrm{Ct})$ of the reference gene and the rep reaction.

\section{Assessment of Plasmid Stability}

The stability of plasmid pRep-q77 containing the proposed

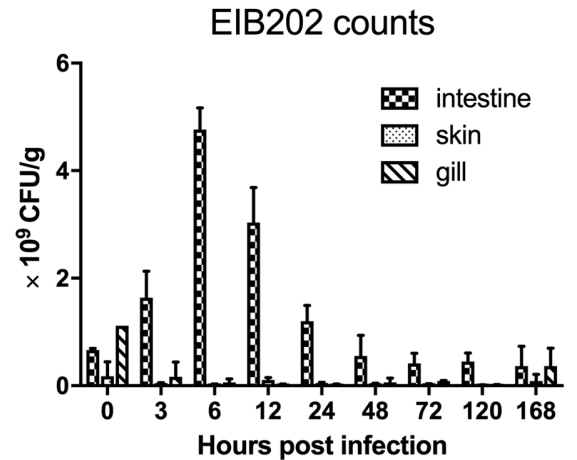

minimal replication region in E. coli and E. piscicida were assessed as follows: E. coli and E. piscicida with pRep-q77 were inoculated in $\mathrm{LB}\left(\mathrm{Cm}^{+}\right)$and grown for $12 \mathrm{~h}$ and $24 \mathrm{~h}$ respectively. Then, the bacterial suspension was taken to antibiotic-free LB at $1 \%(\mathrm{v} / \mathrm{v})$ ratio. The E. coli cells were collected at $0,3,6,9,12,20,24 \mathrm{~h}$, and E. piscicida cells were collected at $0,8,16,24 \mathrm{~h}$ after inoculation. Harvested E. coli and E. piscicida were then serially diluted and inoculated on plates with and without $\mathrm{Cm}$. The curves describing the plasmid loss were drawn as the ratio of counts of colonies on antibiotic-containing plates to antibiotic-free plates.

\section{Immersion Infection of Zebrafish, Sampling and qPCR}

E. piscicida EIB202, ApEIB202/EIB202 and WED were processed as described previously with modifications [18]. The bacteria were harvested, washed and then suspended with sterile PBS at $10^{8} \mathrm{CFU} / \mathrm{ml}$. E. piscicida-free zebrafish were bath-infected with bacterial suspension for $30 \mathrm{~min}$, then washed with clean water, and returned to original tanks. Intestines, skin, and gills of ten zebrafish were pooled and weighed at $0,3,6,12,24,48,72,120$, $168 \mathrm{~h}$ post infection. Total DNA from zebrafish tissue was extracted by using a TIANamp Marine Animals DNA Kit (DP324; TIANGEN, China). Quantitative PCR assays were performed as described previously [19]. Colonization on tissues was determined by bacterial load per gram tissue.

\section{Results}

\section{Role of pEIB202 in Zebrafish Colonization}

We previously developed a live attenuated vaccine called WED, which can effectively protect turbots against edwardsiellosis via intraperitoneal injection. However, the relative percent survival (RPS) in the turbot bath vaccinated with WED was lower than that of vaccination via intraperitoneal injection, which exerted an adverse impact on the wide use of the vaccine. So much work has been done on the development of immersion vaccines, including analysis of the counts of bacteria colonized on

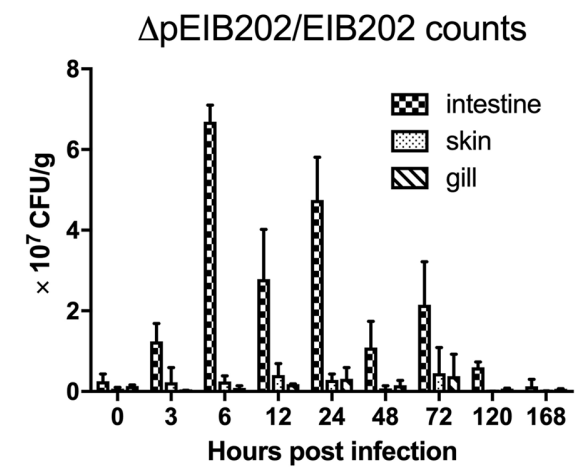

Fig. 1. The counts of Edwardsiella piscicida colonized on intestine, skin, and gills of zebrafish after exposure to $10^{8} \mathrm{CFU} / \mathrm{ml}$ bacteria. Total DNA of each tissue from ten zebrafish was extracted for qPCR analysis. Bars represented the mean counts of three biological replicates and the error bars represented standard deviations. 
zebrafish by qPCR. Mucosal tissues were obtained after zebrafish bath-infection or bath-vaccination with $10^{8} \mathrm{CFU} / \mathrm{ml}$ bacteria. qPCR was performed using E. piscicida specific primers. The result shows that E. piscicida mainly colonize on intestines, which suggests intestine is the main route for E. piscicida infection. The counts of E. piscicida on the intestine peaked at $6 \mathrm{~h}$ post infection, and then decreased to the level at initial infection. The counts using WED are too low to be detected (data not shown). Then we tested the $\Delta$ pEIB202 strain, which colonizes on the intestine at a level 100 times lower than that of E. piscicida EIB202 during a week post infection (Fig. 1). These results indicate that pEIB202 may play an important role in zebrafish colonization.

\section{Location of the Replicon of pEIB202}

Based on the whole sequence of pEIB202 submitted to GenBank (Accession No. CP001136) [12], the plasmid carries 53 predicted ORFs, which confers the multiple functions, of the bacteria such as antibiotic resistance, conjugation, and transposon. Furthermore, pEIB202 encodes an incomplete type IV secretion system, which is involved in horizontal DNA transfer. There is more than one copy of pEIB202 in E. piscicida EIB202, but the origin of replication has not been elucidated. It is necessary to determine the replicon of pEIB202 for characterizing the virulent plasmid. To this end, Sau3A I partial digestion products were screened: 43 transformants were obtained and sequenced, among which the No. 33 transformant contained the minimal Sau3A I DNA fragment of pRep33 (from 18,249 to 21,335 bp). Subsequently, PCR was performed as shown in Fig. 2 and 5 fragments were succeeded. A shorter fragment has been though it unfortunately failed to replicate in E. coli JM83.
Therefore, we located the minimal replication origin from 18,837 to $20,816 \mathrm{bp}$, and named this subclone pRep-q77/ JM83.

Copy counts of these subclones were measured through qPCR (Fig. 2). Results showed that these plasmids have low copy counts in $\Delta$ pEIB202/EIB202 and JM83. Copy counts in JM83 are lower than those in $\Delta$ pEIB202/EIB202, which may be attributed to the uncoordinated operation of the replication apparatus between pEIB202 and E. coli. The relative copy counts of pRep33 (36) and pRep19 (4.7) are the highest among five subclones in $\Delta$ pEIB202/EIB202 and JM83 respectively. Interestingly, pRep13, pRep19, and pRep23 contain the same fragment as pRep33, while having lower copy numbers than pRep33. The differences among them have been carefully checked and a fragment ranging from 18,134 to 18,249 has been found missing in pRep33. In this $116 \mathrm{bp}$ fragment, there are 2 different direct repeats shown in a supplementary Fig., which may regulate the copy number of the subclone. Direct repeats participate in plasmid copy number controlling, by means of cooperation with initiation protein. Moreover the mechanisms of direct repeats to negatively regulate plasmid copy number have been elucidated, one of which is a titration model [20]. Briefly, the higher concentration of direct repeats leads to fewer plasmid copies, due to the competition for a finite amount of initiation protein between parent and daughter plasmid copies. Here, we propose that the high copy number of pRep33 is due to the absence of those two direct repeats, which present in pRep23, pRep19, and pRep13. In conclusion, there are several differences between replication mechanisms involved in $\Delta$ pEIB202/EIB202 and JM83.

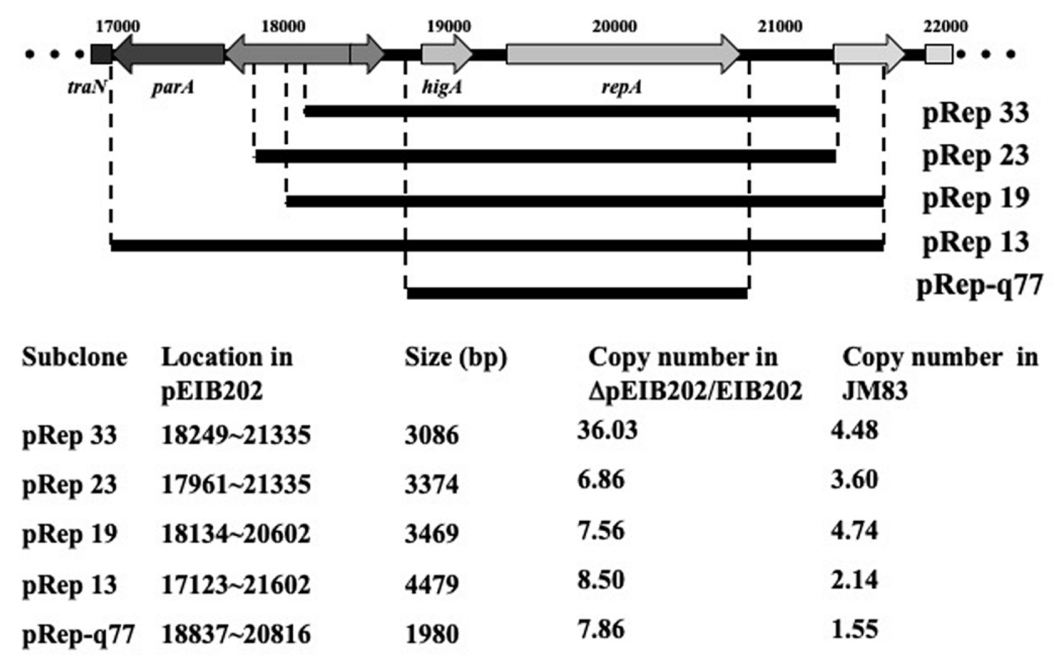

Fig. 2. Relative locations, sizes and copy numbers both in $\Delta$ pEIB202/ E. piscicida EIB202 and E. coli JM83 of the 5 subclones. 


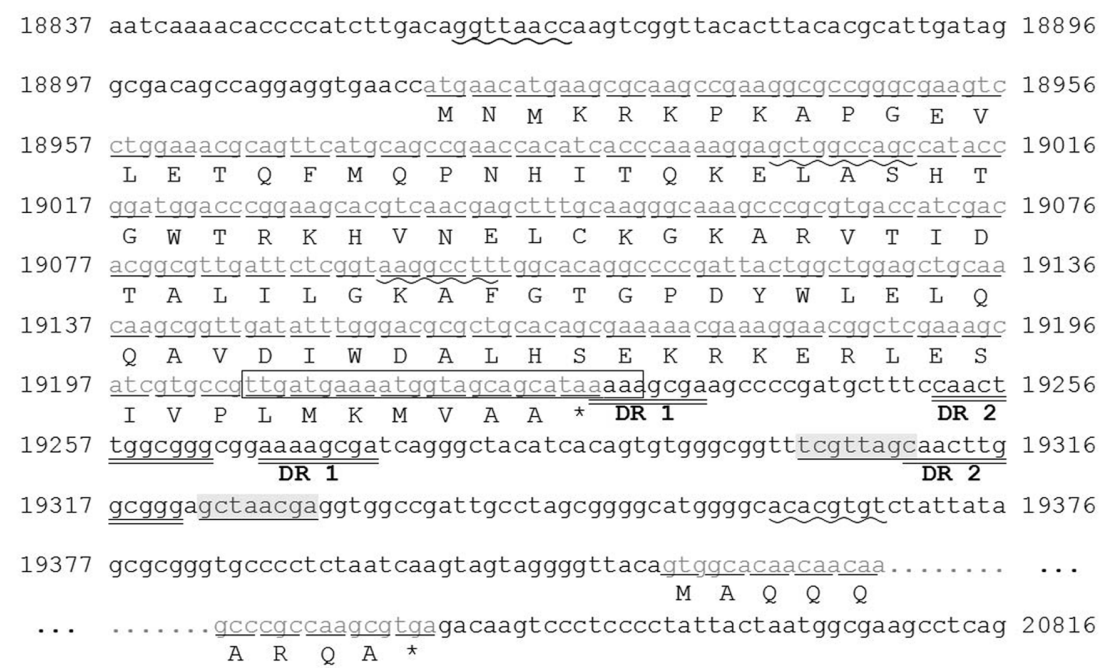

Fig. 3. Sequence of the origin of replication of pEIB202.

The numbers refer to the plasmid position. Inverted repeats (IR) are underlined and shadowed and palindromes are underlined with curved lines. Direct repeats (DR) are double underlined. Also the AT-rich region is boxed. The putative ORFs are shown in gray color, and sequences between 18918 and 19229 bp are predicted to be the transcriptional regulator, while sequences after 19414 encode the putative replication protein.

\section{Sequence Features of Minimal Replication Origin}

The minimal replication origin in plasmid pRep-q77 is located between 18,837 and $20,816 \mathrm{bp}$, containing a transcriptional repressor encoding gene and repA gene. Inverted repeats, direct repeats, and palindrome sequences in this region were screened by DNASTAR (Fig. 3), and an AT-rich region was predicted by mEMBOSS. The direct repeats are viewed as the binding sites for replication protein [21] and play a part in plasmid incompatibility [22], while the inverted repeats are regarded as the interaction region with the HTH motif [23] of replication protein dimer. The AT-rich region is agreed to be an initial part of replication due to poor stability [24].

The transcriptional repressor, consisting of 103 aa, shares

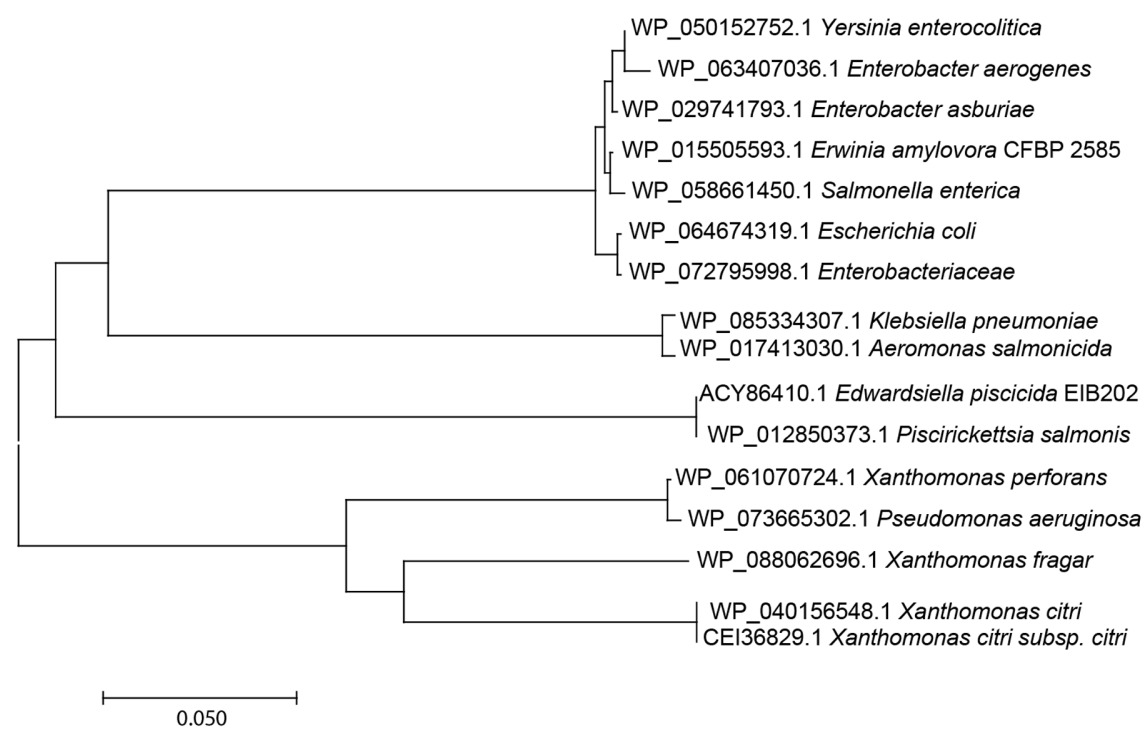

Fig. 4. The phylogenetic tree is constructed with the MEGA 7 using proteins obtained from the Genbank protein database. Top 15 hits for RepA protein sequences from pEIB202 after BLASTP similarity searches are clustered, and the relationships among the proteins are determined. The bar indicates the number of amino acid substitutions per site. 
high amino acid sequence similarity to the putative antidote protein of the plasmid maintenance system (ZP_00107635.1) from Nostoc punctiforme PCC 73102 (identity: 39\%, coverage: $6 \sim 98$ aa). It belongs to the antidote protein HigA family, which forms a distinct group of helix-turn-helix proteins. The HigB/HigA toxin-antitoxin system of E. coli CFT073 participates in many physiological activities, including cell growth and maintenance of plasmid DNA [25]. HigB is a sequence-specific endoribonuclease, which contacts to the $50 S$ ribosomal subunit. The antitoxin HigA shows an interrelated format of shuffling with altered antitoxin folds to neutralize toxins. Thus, we believe that the transcriptional repressor is likely to contribute to plasmid maintenance.

RepA constitutes 454 aa, among which there is a $\operatorname{RepB}$ primase domain between 130 and 220 aa. The conserved motif between 361 and 432 aa shows 31\% identity with the HTH in the C termini of ColE2-P9 Rep. Notably, ColE2-P9 Rep protein is reported as a single protein for DNA unwinding [26], and the HTH module exclusively binds to Ori, which is critical for Rep to anchor on the DNA [27]. Hence, the RepA of pEIB202 is able to unwind the plasmid duplex DNA independently. Besides, the amino acid sequence shows $100 \%$ identity with replication proteins of Piscirickettsia salmonis AY6532B, AY3800B, AY6297, and AY3864B (100\% coverage). Furthermore, RepA presents high similarity with the replication proteins of Yersinia enterocolitica, Erwinia amylovora CFBP, Salmonella enterica, and E. coli (identity: 73\%, coverage: $98 \%$ ). The phylogenetic NJ tree based on RepA is shown in Fig. 4. RepA exhibits a close evolution relationship with hypothetical replication proteins of the pathogenic bacteria, indicating the transfer/ evolution traces of pEIB202.

\section{Stability of pRep-q77}

The stability of pRep-q77 in E. coli JM83 and $\Delta$ pEIB202 EIB202 is assessed. After 24-hour antibiotics-free culturing, $73 \% \Delta$ pEIB202 EIB202 carried the plasmid pRep-q77 and only 25\% E. coli JM83 carried plasmid pRep-q77 (Fig. 5). The result proves that plasmid pRep-q77 is more stable in E. piscicida than in E. coli.

\section{Discussion}

In this study, the minimal replication region of the virulence plasmid pEIB202 is located at a 1,980 bp DNA fragment ranged from $18,837 \mathrm{bp}$ to $20,816 \mathrm{bp}$ and characterized in E. piscicida.

Mucosal tissues (intestines, skin and gills) of fish are

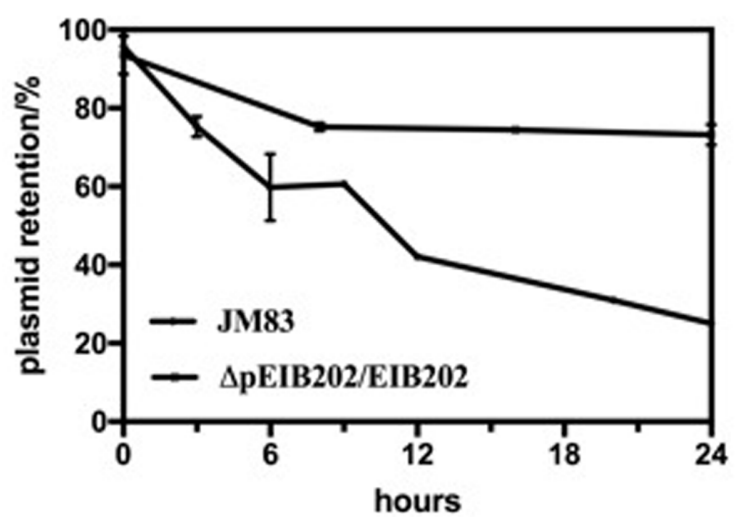

Fig. 5. The plasmid loss rate of pRep-q77 in $\Delta$ pEIB202/ E. piscicida EIB202 and E. coli JM83 during 24 h culturing.

possible accesses for pathogen invasion [28]. Previous study reported that the effectiveness of bath vaccination is related to the amount of antigen uptake [29]. To elucidate genetic factors in E. piscicida EIB202 contributing to the immunoprotection of WED via immersion, we measured the bacteria loads on main mucosal tissues of zebrafish after bath vaccination via qPCR. According to the results, pathogen colonized on the intestine exhibit a significant proliferation at $6 \mathrm{~h}$ post infection, after which bacteria are cleared by the host. The wild-type E. piscicida EIB202 is proved to colonize on the intestine at a level 100 times higher than $\Delta$ pEIB202/EIB202, suggesting the plasmid may play a vital role in colonization. However, the plasmid has several copies in E. piscicida EIB202, impeding further study about its function due to the difficulty of deleting specific genes on pEIB202. Thus, it is necessary to figure out the replication region of pEIB202, although the replicon of pEIB202 was predicted by bioinformatics [12]. Here, the replication region is found to be located in a 1,980 bp region, containing the replication protein RepA and a transcriptional repressor encoding gene. Generally, the replication by the theta type mechanism is more common in gram-negative bacteria. Furthermore, the plasmidencoded replication protein Rep is required [21], implying that pEIB202 exerts the theta mechanism. In addition to Rep protein, general features like an adjacent AT-rich region containing sequence repeats, the $d n a A$ boxes for host DnaA initiator protein binding [30], and the Dam methylation sequences [31] are found in numerous thetareplicating plasmids' original region. Methylation may help to improve the efficiency of replication initiation both in vivo and in vitro. Though it is unnecessary for 
replication, it primarily matters in post-replication [32]. Furthermore, it was reported that methylation sites in the replicon of plasmid P1 are required to sequester hemimethylated oriC into the membrane [33]. The stimulation can result from the increased twist or unwinding of the DNA or accumulated recycling of initiators [34]. This may explain the differences of the copy counts between E. coli and E. piscicida. As the sequence exhibits subtypical features, such as no typical E. coli $d n a A$ boxes [35] and methylation sequences or few inverted repeats, the novel replicon is difficult to predict. Also, E. piscicida specific replication features should be noted.

The RepA shows a close relationship with replication protein of various pathogens including aquatic gammaproteobacteria pathogen P. salmonis. In addition, the plasmid carries genes encoding DNA transfer proteins, implying that the plasmid is able to transfer among different species and confer them multidrug resistance. Maintenance and replication of plasmid may induce a "metabolic burden" in E. coli. It is clarified as the amount of resources (raw material and energy) that are obtained from the host cell metabolism [36]. In E. coli $\mathrm{DH} 5 \alpha$, gene gapA encoding glyceraldehyde-phosphate dehydrogenase (GAPDH) in the glycolytic pathway is observed to be up-regulated in plasmid-bearing cells [37]. The expression of $f b a$ gene (encoding fructose bisphophate aldolase) in E. coli BL21(DE3) is increased as well [38]. Those demonstrate that house keeping genes for energy metabolism are likely to upregulate due to the plasmid introduction, which can be attributed to "metabolic burden" brought from plasmid. In addition, GAPDH and FBA are found at the noncytoplasmic parts, and present protection against several pathogens in turbots [39, 40], suggesting these house keeping genes act as virulent factors when secreted. This suggests that pEIB202 may confer up-regulation of some genes, such as GAPDH and FBA in E. piscicida EIB202, hence its potential virulent-related effects on E. piscicida EIB202.

\section{Acknowledgments}

This work was financially supported by grants from the National Natural Science Funds of China (31372550).

\section{Conflict of Interest}

The authors have no financial conflicts of interest to declare.

\section{References}

1. Park SB, Aoki T, Jung TS. 2012. Pathogenesis of and strategies for preventing Edwardsiella tarda infection in fish. Vet. Res. 43: 67.

2. Miyazaki T, Kaige N. 1985. Comparative histopathology of edwardsiellosis in Fishes. Fish Pathol. 20: 219-227.

3. Mohanty BR, Sahoo PK. 2007. Edwardsiellosis in fish: a brief review. J. Biosci. 32: 1331-1344.

4. Xu T, Zhang XH. 2014. Edwardsiella tarda: An intriguing problem in aquaculture. Aquaculture 431: 129-135.

5. Rijkers GT, Teunissen AG, Van Oosterom R, et al. 1980. The immune system of cyprinid fish. The immunosuppressive effect of the antibiotic oxytetracycline in carp (Cyprinus Carpio L.). Aquac. 19: 177-189.

6. Xu T, Su Y, Xu Y, et al. 2014. Mutations of flagellar genes Flic12, Flia and Flhdc of Edwardsiella tarda attenuated bacterial motility, biofilm formation and virulence to fish. $J$. Appl. Microbiol. 116: 236-244.

7. Dong X, Fan X, Wang B, et al. 2013. Invasin of Edwardsiella tarda is essential for its haemolytic activity, biofilm formation and virulence towards fish. J. Appl. Microbiol. 115: 12-19.

8. Xiao J, Chen T, Wang Q, Zhang Y. 2012. Comparative analysis of the roles of catalases KatB and KatG in the physiological fitness and pathogenesis of fish pathogen Edwardsiella tarda. Lett. Appl. Microbiol. 54: 425-432.

9. Xiao J, Chen T, Yang M, Zhang Y, Wang Q. 2012. Identification of qseEGF genetic locus and its roles in controlling hemolytic activity and invasion in fish pathogen Edwardsiella tarda. Lett. Appl. Microbiol. 55: 91-98.

10. Wang X, Wang Q, Yang M, Xiao J, Liu Q, Wu H, et al. 2011. QseBC controls flagellar motility, fimbrial hemagglutination and intracellular virulence in fish pathogen Edwardsiella tarda. Fish Shellfish Immunol. 30: 944-953.

11. Xiao $\mathrm{J}^{1}$, Chen T, Liu B, Yang W, Wang Q, Qu J, et al. 2013. Edwardsiella tarda mutant disrupted in type III secretion system and chorismic acid synthesis and cured of a plasmid as a live attenuated vaccine in turbot. Fish Shellfish Immunol. 35: 632-641.

12. Wang Q, Yang M, Xiao J, Wu H, Wang X, Lv Y, et al. 2009. Genome sequence of the versatile fish pathogen Edwardsiella tarda provides insights into its adaptation to broad host ranges and intracellular niches. PLoS One 4: e7646.

13. Jingfan Xiao, QiyaoWang, Qin Liu, XinWang, Huan Liu, Yuanxing Zhang, et al. 2008. Isolation and identification of fish pathogen Edwardsiella tarda from mariculture in China. Aquac. Res. 40: 13-17.

14. Wang X, Wang Q, Xiao J, Liu Q, Wu H, Xu L, et al. 2009. Edwardsiella tarda T6SS component evpP is regulated by esr $B$ and iron, and plays essential roles in the invasion of fish. Fish Shellfish Immunol. 27: 469-477.

15. Xi X, Fan J, Hou Y, Gu J, Shen W, Li Z, Cui Z. 2013. Characterization of three cryptic plasmids from Lactobacillus 
plantarum G63 that was isolated from Chinese pickle. Plasmid. 70: 321-328.

16. Lee CL, Ow DS, Oh SK. 2006. Quantitative real-time polymerase chain reaction for determination of plasmid copy number in bacteria. J. Microbiol. Methods 65: 258-267.

17. Xie G, Huang J, Zhang Q, et al. 2012. A real-time PCR targeted to the upstream regions of $\mathrm{HlyB}$ for specific detection of Edwardsiella tarda. Chin. J. Oceanol. Limnol. 30: 731-737.

18. Liu X, Wu H, Chang X, Tang Y, Liu Q, Zhang Y. 2014. Notable mucosal immune responses induced in the intestine of zebrafish (Danio Rerio) bath-vaccinated with a live attenuated Vibrio anguillarum vaccine. Fish Shellfish Immunol. 40: 99-108.

19. Liu X, Chang $X$, Wu H, Xiao J, Gao Y, Zhang Y. 2014. Role of intestinal inflammation in predisposition of Edwardsiella tarda infection in zebrafish (Danio Rerio). Fish Shellfish Immunol. 41: 271-278.

20. Dhruba KC, Thomas DS. 1997. Replication control of plasmid P1 and its host chromosome: the common ground. Prog. Nucleic Acid Res. Mol. Biol. 57: 145-8.

21. del Solar G, Giraldo R, Ruiz-Echevarría MJ, Espinosa M, Díaz-Orejas R. 1998. Replication and control of circular bacterial plasmids. Microbiol. Mol. Biol. Rev. 62: 434-464.

22. Novick RP. 1987. Plasmid incompatibility. Microbiol Rev. 51: 381-395.

23. De Viedma DG, Serrano-Loópez A, Diaz-Orejas R. 1995. Specific binding of the replication protein of plasmid pPS10 to direct and inverted repeats is mediated by an HTH motif. Nucleic Acids Res. 23: 5048-5054.

24. Skarstad K, Baker TA, Kornberg A. 1990. Strand separation required for initiation of replication at the chromosomal origin of $E$. coli is facilitated by a distant RNA--DNA hybrid. EMBO J. 9: 2341-2348.

25. Arbing MA, Handelman SK, Kuzin AP, Verdon G, Wang C, Su M, et al. 2010. Crystal structures of Phd-Doc, HigA, and YeeU establish multiple evolutionary links between microbial growth-regulating toxin-antitoxin systems. Structure 18: 9961010.

26. Itou H, Yagura M, Shirakihara Y, Itoh T. 2015. Structural basis for replication origin unwinding by an initiator primase of plasmid ColE2-P9: duplex DNA unwinding by a single protein. J. Biol. Chem. 290: 3601-3611.

27. Yagura M, Nishio SY, Kurozumi H, Wang CF, Itoh T. 2006. Anatomy of the replication origin of plasmid ColE2-P9. J. Bacteriol. 188: 999-1010.
28. Corrales J, Mulero I, Mulero V, Noga EJ. 2010. Detection of antimicrobial peptides related to piscidin 4 in important aquacultured fish. Dev. Comp. Immunol. 34: 331-343.

29. Bordas MA, Balebona MC, Rodriguez-Maroto JM, Borrego JJ, Morinigo MA. 1998. Chemotaxis of pathogenic Vibrio Strains towards mucus durfaces of Gilt-Head Sea Bream (Sparus Auratal.). Appl. Environ. Microbiol. 64: 1573-1575.

30. Bramhill D, Kornberg A. 1988. A model for initiation at origins of DNA replication. Cell 54: 915-918.

31. Brendler TG, Abeles AL, Reaves LD, Austin SJ. 1991. Unique sequence requirements for the P1 plasmid replication origin. Res. Microbiol. 142: 209-216.

32. Abeles A, Brendler T, Austin S. 1993. Evidence of two levels of control of P1 OriR and host OriC replication origins by DNA adenine methylation. J. Bacteriol. 175: 7801-7807.

33. Brendler T, Abeles A, Austin S. 1995. A protein that binds to the P1 origin core and the OricC 13 mer region in a methylation-specific fashion is the product of the host SeqA gene. EMBO J. 14: 4083.

34. Chattoraj DK, Schneider TD. 1997. Replication control of plasmid PI and its host chromosome: The Common ground. Prog. Nucleic Acid Res. Mol. Biol. 57: 145-152.

35. Schaefer C, Messer W. 1991. DnaA protein/DNA interaction. Modulation of the recognition sequence. Mol. Gen. Genet. 226: 34-40.

36. Bentley WE, Kompala DS. 1990. Optimal induction of protein synthesis in recombinant bacterial culturesa. Ann. NY Acad. Sci. 589: 121-138.

37. Ow D S-W, Nissom PM, Philp R, Oh S.KW, Yap M G-S. 2006. Global transcriptional analysis of metabolic burden due to plasmid maintenance in Escherichia coli DH5 $\alpha$ during batch fermentation. Enzyme Microb. Technol. 39: 391-398.

38. Wang Z, Xiang L, Shao J, Wegrzyn A, Wegrzyn G. 2006. Effects of the presence of ColE1 plasmid DNA in Escherichia coli on the host cell metabolism. Microb. Cell Fact. 5: 34.

39. Liang S, Wu H, Liu B, Xiao J, Wang Q, Zhang Y. 2012. Immune response of turbot (Scophthalmus Maximus L.) to a broad spectrum vaccine candidate, recombinant glyceraldehyde3-phosphate dehydrogenase of Edwardsiella tarda. Vet. Immunol. Immunopathol. 150: 198-205.

40. Sun Z, Shen B, Wu H, Zhou X, Wang Q, Xiao J, Zhang Y. 2015. The secreted fructose 1,6-bisphosphate aldolase as a broad spectrum vaccine candidate against pathogenic bacteria in aquaculture. Fish Shellfish Immunol. 46: 638-647. 